\title{
Estudio limnológico para determinar la calidad del agua en cuerpos lóticos. Metodología
}

María Teresa Ortiz V.

\section{Resumen}

El aftioulo explica un procedimiento senoilto para la realización de estudios timnołógicos de cuerpos de agua, preferentemente lóticos, qunque también puede ser qplicado a cuerpos de agua lénticos, para determinar su calidad y posibles usos en proyectos agroecológicos o estudios de impacto ambiental.

El procedimiento consta de seis pasos, descritos con base en estudios realizados con anterioridad por la qutora. Además, se recupera una muestra fotográfica de la fauna bentónica del Río Pasto, con su correspondiente olasificación taxonómica. (Anexo)

El procedimiento tiene su aplicación principalmente en corrientes medias y de baja protundidad. Además es de especial importancia pedagógica, foda vez que permite comprender las reldciones de los organismos hidrobiológioos, dentro de un ecosistema coútico y su repercusión en todo el entomo. Asímismo, proporciona un enfoque limnológico, empleado en el estudio de cuencas hidrográficas, pero que podría servir de modelo dentro de estudios de cuerpos de agua similares.

\section{Palabras clave}

Limnología, Bentos, Lófico, Léntico, Parámetros hidrobiológicos.

\begin{abstract}
The article explains a simple procedure for imnologicat studies, preferably for totic waters. although it can aiso be apolied to lentio waters.

The procedure consists of six steps that are described on the basis of studies conducted earlier by the quthor. In addifion, it provides a photographic exhibition of the benthio fauna in the iver Pasto with its toxonomio classification.

The procedure has its application principally in average currents and of low depth. Besides it is particularly important from the pedagogioal point of view, since it allows understand the relevance of relations between different hydrobiological agents within an aquatic ecosystem and its impact on the entire environment. It aiso provides an limnological approcoh that coutd be both applied for studying watersheds, and as a modet in studies of similar bodies of water.
\end{abstract}

\section{Key words}

Limnology, Benthos, Lotic, Lentic, Hydrobiological parameters. 


\section{Introducción}

La limnología parte de un estudio de los fenómenos físico-químicos y biológicos de aguas continentales, epicontinentales, fluyentes o estancadas, con base en el cual se puede determinar el estado de un ecosistema. (Margalef, 1983)

El uso de procedimientos limnológicos permite evaluar integralmente la calidad del agua, al tiempo que proporciona una visión sistémica del estado de alteración que presenta el agroecosistema. Los contaminantes generados por las actividades agropecuarias y que llegan al agua por diferentes vías, demandan un trabajo ecológico armónico y permanente, orientado a lograr la sostenibilidad del medio ambiente.

Los estudios limnológicos cobran cada vez mayor importancia práctica, porque ofrecen información fundamental en la determinación de la calidad de los cuerpos de agua. Esto en virtud de que uno de los elementos principales en la calidad hídrica es, sin duda, la identificación de los organismos hidrobiológicos que en un cuerpo de agua, o más ampliamente en un sistema hidrográfico, coexisten. Sin embargo, a pesar de la información que aporta en estos términos la limnología, son pocos los que aplican su enfoque al momento de evaluar la calidad o capacidad de vida de las aguas; más bien, se emplea una serie de indicadores que, por no comprometer un análisis a fondo de los indicadores biológicos y su balance ecológico, sólo ofrecen un cuadro parcial e impreciso de la vitalidad del sistema.

Los estudios limnológicos proveen toda la información, necesaria para realizar planes de mejoramiento de un ecosistema acuático que repercutirán en la totalidad del ecosistema. De allí parte la importancia de conocer la forma en que se realiza un estudio limnológico, teniendo en cuenta una serie de procedimientos o pasos, que permitirán obtener un análisis sistémico y obtener conclusiones, que proporcionen la base para un plan de mejoramiento ambiental.

Entendiendo que el agua es el elemento fundamental de la vida y el mejor indicador de la calidad de un ecosistema; requiere un cuidado especial, que incluye la creación de estrategias de protección y saneamiento, llevando a cabo programas de reglamentación de vertimientos que pueden provenir de desechos sanitarios humanos (contaminación puntual), de actividades antropogenias, piscicultura, minería, industrial (contaminación difusa) o incluso de problemas inherentes a la composición química del agua.
Puede notarse en los estudios agroecológicos la ausencia del análisis de ecosistemas, desde la perspectiva de la calidad del agua, situación que lleva a proponer este enfoque como mecanismo para la determinación y mejoramiento de un agroecosistema, a partir de la caracterización físico-química, biológica y bacteriológica de los cuerpos de agua.

\section{Metodología}

El presente artículo se basa en la descripción de la forma de realización de un estudio limnológico. Los datos aquí consignados provienen de la experiencia del autor en la ejecución de estudios limnológicos, entre los cuales es posible citar: a) Estudio limnológico del Río Pasto y sus principales afluentes; b) Estudio limnológico del Río Guamués; y, c) Estudio limnológico del Río Bobo.

La metodología seguida partió de la recolección de los diferentes estudios, que luego fueron comparados, para identificar los procesos similares y las etapas de los mismos. Por tratarse de una recolección de datos secundarios, el artículo se estructura a partir de métodos cualitativos como la observación directa y la consulta bibliográfica.

El método cualitativo más utilizado es inductivo y descriptivo por observación; se infirieron ciertas secuencias lógicas de estudios, los cuales otorgan un punto de partida para su posterior profundización. (Ortíz, 1995) (Wetzel, 2000)

\section{Procedimiento para un estudio limnológico}

Las etapas o procedimientos principales de todo estudio limnológico pueden agruparse inicialmente en seis pasos, abiertos a discusión. Estos pasos se basan en la experiencia adquirida en la realización de dichos estudios, y se ponen de manifiesto en este artículo, para que sirvan de guía a quienes deseen realizar estudios de este tipo.

Los pasos a seguir son:

Establecimiento de la red de muestreo.

Características de los hábitats en las estaciones de muestreo.

Selección de los parámetros físico-químicos, bacteriológicos y biológicos de la calidad de agua.

Método para la toma de muestras.

Análisis de laboratorio.

Discusión de los resultados.

En adelante, se tratará de detallar lo que significa cada uno de ellos. 


\section{Establecimiento de la red de muestreo}

Es el primer paso del estudio limnológico y consiste en determinar el área y secuencia de lugares en los cuales se tomarán las muestras. Para este punto es importante detallar la longitud o extensión del cuerpo de agua (río, laguna, lago, entre otros), sus límites y el sentido en el que se tomarán las muestras (por ejemplo, desde el nacimiento del río hasta su desembocaduraj, así como caracterizar la presión doméstica, agrícola e industrial, lo cual, a su vez puede llevar a realizar otro tipo de segmentación. (Ortíz, 1995)

También se debe especificar la segmentación de la corriente de forma cualitativa (contaminación por basuras, descargas de colectores municipales, alcantarillas de industrias, limpieza de establos, químicos de los cultivos, entre otros) y cuantitativa (volúmenes de los diferentes tipos de contaminantes que pueden ser arrojados al cuerpo de agua).

Para la segmentación del río, los segmentos a analizar deben incluir estaciones de muestreo y principales afluentes, como lugares mínimos para el levantamiento de información. Antes de segmentar el río debe realizarse un análisis previo del lugar, con el propósito de determinar las estaciones representativas de cada sector.

Para seleccionar las corrientes que se someterán a muestreo se debe analizar la sectorización hídrica; esto es, la división de cada cuenca en sus correspondientes subcuencas y estas, a su vez, en las microcuencas que las conforman.

Realizado lo anterior, se procede a escoger las corrientes más representativas para ser sometidas a muestreo, y de este modo lograr una visión más completa del entorno que esté exenta de imprecisiones para la caracterización fisicoquímica, bacteriológica y biológica de todo el río.

\section{Caracterísficas del hábitat de las estaciones de muestreo}

Tras realizar la segmentación, se procede a caracterizar cada uno de los segmentos definidos, teniendo en cuenta que deben ser representativos. En caso tal que se presenten cambios durante la investigación en los segmentos representativos, tales como descargas contaminantes o cambios en las condiciones climáticas, hidráulicas, químicas o físicas del ecosistema, debe definirse un nuevo segmento. (Wetzel, 2000)

Entre los parámetros que deben definirse para cada uno de los segmentos, pueden anotarse:

Longitud (m)
Velocidad promedio $(\mathrm{m} / \mathrm{seg}$.)

Caudal promedio $\left(\mathrm{m}^{3} / \mathrm{seg}\right.$.)

Ancho de la sección de muestreo (m)

Profundidad de la sección de muestreo $(\mathrm{cm})$

Área promedio $\left(\mathrm{m}^{2}\right)$

Pendiente del terreno (\%)

Las corrientes de agua seleccionadas, deben responder a criterios como caudal, grado de contaminación, longitud y uso por parte de la población beneficiaria. Para evaluar estos criterios se debe establecer una red de muestreo que incluya los siguientes puntos:

Punto de referencia: es necesario fijar sectores representativos de determinados tramos. Estos sectores deben estar libres de estrés ecológico y deben encontrarse aislados de contaminantes y de cualquier riesgo potencial de impacto negativo, pues servirán de referencia.

Sector de soporte: es un tramo o sector en el que se identifica estrés, y que, por tal razón será el soporte del estudio.

Sector de observación: puede ser uno o varios, que se destinarán para la observación de consecuencias de descargas contaminantes, y que posteriormente servirán para identificar la evolución del proceso de autodepuración del agua.

Sector de control: por último, se definirá un sector inferior o tramo, el cual servirá de punto de control de la capacidad de autodepuración del curso de agua. El control debe realizarse teniendo en cuenta los factores contaminantes y el caudal de la pendiente en función, entre otras características fisicoquímicas del recorrido del agua, analizando en cada uno de estos factores su naturaleza e importancia o incidencia en la calidad del ecosistema.

ver figura 7 en la siguiente páging

Selección de parámetros físico-químicos, bacteriológicos y biológicos para análisis de la calidad de agua

Los parámetros físicos incluyen temperatura, color, olor turbiedad, sólidos (totales, volátiles, suspendidos, disueltos y sedimentables).

Los parámetros químicos escogidos son oxígeno disuelto, demanda bioquímica de oxígeno (DBO), demanda química de oxígeno (DQO) y pH.

Los parámetros bacteriológicos incluyen el número más probable de coliformes totales y coliformes fecales (NMCT y NMCF). 
Ficha No. (_) (Defina el número de la Ficha)

\begin{tabular}{|l|l|}
\hline ESTACIÓN No. & $1,2,3,4,5,6$ eto. \\
\hline $\begin{array}{l}\text { FECHA Y HORA DE } \\
\text { MUESTREO }\end{array}$ & EJ: NACIMIENTO DEL RIO \\
\hline NOMBRE DE LA ESTACIÓN & NOMBRE DEL LUGAR \\
\hline LUGAR DE MUESTREO & $\begin{array}{l}\text { Ej.: } \\
\text { Estrés (libre o contaminado) } \\
\text { Tipo de sustratoCorriente } \\
\text { Tipo de vegetación en el río } \\
\text { Tipo de vegetación en la orilla } \\
\text { Presencia de organismos bentónicos } \\
\text { Lista cualitativa de especies de macro fauna } \\
\text { Presente } \\
\text { Tipo de orilla. }\end{array}$ \\
\hline LARACTERÍSTICAS DEL HÁBITTES & $\begin{array}{l}\text { Ejomplo: COMPRENDIDO ENTRE EL NACIMIENTO, EN } \\
\text { EL. PÁRAMO Y LA CONVERGENCIA DE LAS GUEBRADAS } \\
\text { A, B Y C }\end{array}$ \\
\hline
\end{tabular}

Figura 1: Ficha de muestreo.

Los parámetros biológicos como el grado de eutrofización del agua y organismos.

La fauna bentónica (zoobentos) en su evaluación cualitativa y cuantitativa permite una clasificación de la calidad del agua desde un parámetro biológico.

Ver figura 2 en la siguiente página

\section{Método para la toma de muestras Toma de muestras de agua}

La caracterización se inicia con la toma de muestras de aguas superficiales para los análisis de los parámetros físico-químicos generales en aguas naturales, para lo cual, se requieren tres litros de muestra, $y$ aparte se realiza el análisis bacteriológico, con base en $300 \mathrm{ml}$ de muestra en frascos esterilizados. Algunos parámetros tales como oxígeno disuelto $(\mathrm{mg} / \mathrm{l})$, conductividad, $\mathrm{pH}$, presión atmosférica (Mbar), temperatura $\left({ }^{\circ} \mathrm{C}\right)$ del agua y del ambiente, caudal (m3/ seg.) y velocidad de la corriente ( $\mathrm{m} / \mathrm{seg}$.), se analizan in situ. (Ortíz, 1995; Rueda, 2002)

\section{Toma de muestras de bentos}

Las muestras de macro fauna bentónica se recogen de sedimentos con una draga Eckman a $10 \mathrm{~cm}$. de profundidad en el fondo del lecho del río. En ocasiones, es necesario remover las piedras del fondo para tomar muestras de los organismos adheridos a las mismas. Posteriormente, se conservan los organismos en frascos con alcohol, para ser clasificados taxonómicamente. A partir del inventario de fauna bentónica en distintos sitios se confrontan indicadores ecológicos de calidad representativos de las ca- racterísticas específicas del hábitat en que son colectadas. Por último, se evalúa la calidad de cada ambiente de acuerdo al número de especies, tipo de organismos y diversidad biológica. (Ortíz, 1995; Rueda, 2002)

\section{Análisis de laboratorio}

Los métodos de análisis de laboratorio pueden ser físico-químicos, bacteriológicos o biológicos.

Los análisis físico-químicos y bacteriológicos se realizan de acuerdo con los métodos reglamentados por la normatividad nacional en materia de calidad de agua para diversos usos. Estos análisis están regidos por el Decreto 2105/1984, Decreto 194/1984, normas y criterios del Título Il de la Ley09 de 1979.

El análisis biológico se realiza a partir de la obtención de muestras, el posterior aislamiento de los organismos zoobentónicos ${ }^{\dagger}$, el análisis por medio de estereoscopio de cada uno de ellos y la clasificación cuantitativa (utilización de claves) y cualitativa (aplicación de índices) que permite determinar la calidad del agua en tres rangos representativos: a) aguas oligotróficas o limpias; b) aguas mesotróficas o medianamente contaminadas; $y_{i}$ c) aguas eutróficas o contaminadas.

Para el cálculo del índice de diversidad de géneros se utiliza la ecuación propuesta por Shannon-Weaver (Cole, 1988):

$$
I D=-\sum \frac{n_{i}}{N} \lg \frac{n_{i}}{N}
$$

En donde:

$\mathrm{N}$ : número total de organismos en la muestra. ni: número de individuos en cada grupo (género). Nt: número de grupos en la muestra (géneros.

\section{Discusión de los resultados}

Luego de tener los resultados de laboratorio, se realiza la discusión de los mismos, teniendo en cuenta los parámetros físico-químicos, biológicos y bacteriológicos antes descritos. A través de una comparación y análisis detallado, se determinan el tipo de ecosistema y la calidad de las aguas, de acuerdo con la Reglamentación del Secretaria de Salud. (Ramírez, 1998)

Ver Anexo fotográfico de los principales especímenes encontrados a lo largo del Río Pasto en 1995 


\begin{tabular}{|c|c|c|c|}
\hline $\begin{array}{c}\text { Tipo de } \\
\text { parámetro }\end{array}$ & Parámetro & Unidad & Definición \\
\hline \multirow{5}{*}{ Parámetros físicos } & Temperatura & ${ }^{\circ} \mathrm{C}$ & $\begin{array}{l}\text { Se incrementa por descargas calientes y por la reac- } \\
\text { ción bioquímica durante la degradación de la ma- } \\
\text { teria orgánico. Se puede medir con teletermómetros } \\
\text { y termómetros. }\end{array}$ \\
\hline & Color & UPC & $\begin{array}{l}\text { Indica la edad de las aguas residuales. Determina el } \\
\text { nivel de taninos y ácidos húmicos provenientes de la } \\
\text { descomposición de la lignina en aguas superficiales. } \\
\text { Color aparente: ocasionado por la materia suspendi- } \\
\text { da.Color verdadero: causado por sustancias vegeta- } \\
\text { les de tipo coloidal y sustancias en solución. }\end{array}$ \\
\hline & Olor & - & $\begin{array}{l}\text { Se producen por descomposición de la materia orgá- } \\
\text { nica. Se asocian con sulfuro de hidrógeno, producto } \\
\text { de la reducción de sulfatos a sulfitos por acción de } \\
\text { microorganismos anaerobios. }\end{array}$ \\
\hline & Turbiedad & NTU & $\begin{array}{l}\text { Mide la capacidad de transmisión de la luz a través } \\
\text { del agua. Las partículas suspendidas (coloidales has- } \\
\text { ta granulares) determinan este parámetro. Dificulta } \\
\text { las operaciones de filtración en los sistemas de trata- } \\
\text { miento. }\end{array}$ \\
\hline & Sólidos & $\mathrm{mg} / \mathrm{l}$ & $\begin{array}{l}\text { Existen orgánicos e inorgánicos. Los tipos de sólidos } \\
\text { son: totales (ST), volátiles (SV), fjos (SF), suspendidos } \\
\text { (SS), disueltos (SD) y sedimentables (SSD). }\end{array}$ \\
\hline \multirow{5}{*}{ Parámetros Químicos } & Oxígeno disuelto & $\mathrm{mg} / \mathrm{l}$ & $\begin{array}{l}\text { Oxígeno disuelto en el agua. La presión atmosférica } \\
\text { y temperatura determinan su solubilidad (contenido } \\
\text { de sales). Méto do Winkler y Oxímetro digital. }\end{array}$ \\
\hline & $\begin{array}{l}\text { Demanda } \\
\text { Bioquímica de } \\
\text { Oxigeno (DBO) }\end{array}$ & $\mathrm{mg} / \mathrm{l}$ & $\begin{array}{l}\text { Oxígeno requerido por las bacterias en los procesos } \\
\text { de descomposición aeróbica. Permite medir el gra- } \\
\text { do de polución de una corriente de agua. Medición } \\
\text { por medio de dicromato de potasio. }\end{array}$ \\
\hline & $\begin{array}{l}\text { Demanda } \\
\text { Química de } \\
\text { Oxigeno (DQO) }\end{array}$ & $\mathrm{mg} / \mathrm{l}$ & $\begin{array}{l}\text { Proporciona una medida de la demanda de oxíge- } \\
\text { no del agua a partir de un agente químico. Es más } \\
\text { rápida y oxida la mayoría de los compuestos orgá- } \\
\text { nicos. }\end{array}$ \\
\hline & $\mathrm{pH}$ & - & $\begin{array}{l}\text { Permite determinar la acidez o alcalinidad del agua } \\
\text { de acuerdo al nivel de concentración del lon hidró- } \\
\text { geno en el agua. Se mide con el pH-metro. }\end{array}$ \\
\hline & $\mathrm{CO} 2$ & $\mathrm{mg} / \mathrm{l}$ & $\begin{array}{l}\text { Gas disuelto en el agua, cuyas concentraciones de- } \\
\text { ben encontrarse entre 0,55y 0,60 mg/l. Se puede me- } \\
\text { dir con Método de la Fenoftaleína. }\end{array}$ \\
\hline $\begin{array}{l}\text { Parámetros } \\
\text { Biológicos }\end{array}$ & Eutrofización & & $\begin{array}{l}\text { Cantidad de biomasa vegetal presente en el agua } \\
\text { producida por el exceso de nutrientes (nitritos, nitra- } \\
\text { tos, fosfatos) que reciben los cuerpos de agua. }\end{array}$ \\
\hline \multirow[t]{2}{*}{$\begin{array}{l}\text { Parámetros } \\
\text { bacteriológicos }\end{array}$} & $\begin{array}{l}\text { Coliformes } \\
\text { totales }\end{array}$ & NMP/dl & Bacterias totales \\
\hline & $\begin{array}{l}\text { Coliformes } \\
\text { fecales }\end{array}$ & NMP/dl & Bacterias de origen intestinal \\
\hline
\end{tabular}

Figura 2. Tabla de parámetros (Coimenar, 2002; Ramírez, 1998) 


\section{Conclusiones}

- Los estudios limnológicos permiten determinar el estado de un ecosistema. A partir de ellos se puede obtener información acerca de la calidad de los cuerpos de agua. El análisis de los indicadores biológicos, el balance ecológico y la identificación de los organismos hidrobiológicos del agua, proporciona una base clara para realizar planes de mejoramiento de los ecosistemas.

- El proceso para realizar un estudio limnológico se organiza en seis etapas, a saber: 1) establecimiento de la red de muestreo; 2) características de los hábitats las estaciones de muestreo; 3) selección de los parámetros físico-químicos y bacteriológicos de la calidad de agua; 4) método para la toma de muestras; 5) análisis de laboratorio; y, 6) discusión de los resultados.

- Estos pasos presentan una secuencia lógica y permiten obtener resultados confiables, a través de la obtención de muestras y el posterior estudio comparativo de los niveles de contaminación en- contrados en los cuerpos de agua, en referencia a estándares de calidad de agua óptimos y a la normatividad vigentes.

\section{Referencias}

[1] Cole, G. (1988). Manual de Limnología. Agropecuaria Hemisferio Sur. Buenos Aires, Argentina.

[2] Colmenar, E. (1 de Octubre de 2002). Un temómetro para las aguas. Recuperado el 30 de 06 de 2008, de Ministerio de Me dio Ambiente, Medio Rurd y Marino (España):http://Nmw.mma. es/secciones/biblioteca_publicacion/publicaciones/revista_ambientain 15/pdf/47 52 limno.pdf

[3] Margalef, R. (1983). Limnología. Barcelona: Omega.

[4] Ortiz, M. T. (1995). Estudio limnológico del Río Pasto y sus principales afluentes, con énfasis en la macrofauna bentónica como indicador biológico de la calidad del agua. Corponariño. Pasto.

[5] Ramírez, A. (1998). Limnología colombiana aportes a su conocimiento y estadísticas de análisis. Bogotá: Fundación Universidad de Bogotá Jorge Tadeo Lozano.

[6] Rueda, G. (2002). Manual de métodos en limnología. Bogotá: Asociación Colombiana de Limnología.

[7] Wetzel, R. (2000). Limnological analyses. New York: Springer.

Maria Teresa Orfiz V. Ph.D. en Biología de la Universidad Estatal de Kiev (Ucrania), 1987. En la actualidad, es docente asociada del Programa de Ingeniería Agroecológica de la Corporación Universitaria Minuto de Dios (UNIMINUTO).

mayteortizv@gmail.com

Anexo fotográfico de los principales especímenes encontrados a lo largo del Río Pasto en 1995

\begin{tabular}{|c|c|c|c|c|}
\hline ORDEN & FAMILIA & GENERO & IND. BIOLÓGICO & FOTO \\
\hline AMPHYPODA & HYALLELIDAE & HYALLELA & $\begin{array}{l}\text { RESISTEN ALTOS } \\
\text { NIVELES DE } \\
\text { CONTAMINACIÓN }\end{array}$ & \\
\hline BASSOMATOPHORA & PHYSIDAE & PHYSA & $\begin{array}{l}\text { OLIGO-MESO- } \\
\text { EUTRÓFICAS }\end{array}$ & \\
\hline COLEÓPERA & ELMIDAE & MACRELMIS & $\begin{array}{l}\text { AGUAS OLIGO A } \\
\text { MESOTRÓFICAS }\end{array}$ & \\
\hline COLEÓPTER & GYRINIDAE & $\begin{array}{c}\text { ANDOGYRUS } \\
\text { OGYRINUS }\end{array}$ & $\begin{array}{c}\text { AGUAS } \\
\text { MESOTRÓFICAS }\end{array}$ & \\
\hline
\end{tabular}

Inventum No. 5 Facultad de Ingeniería Uniminuto - Diciembre de 2008 - ISSN 1909 - 2520 


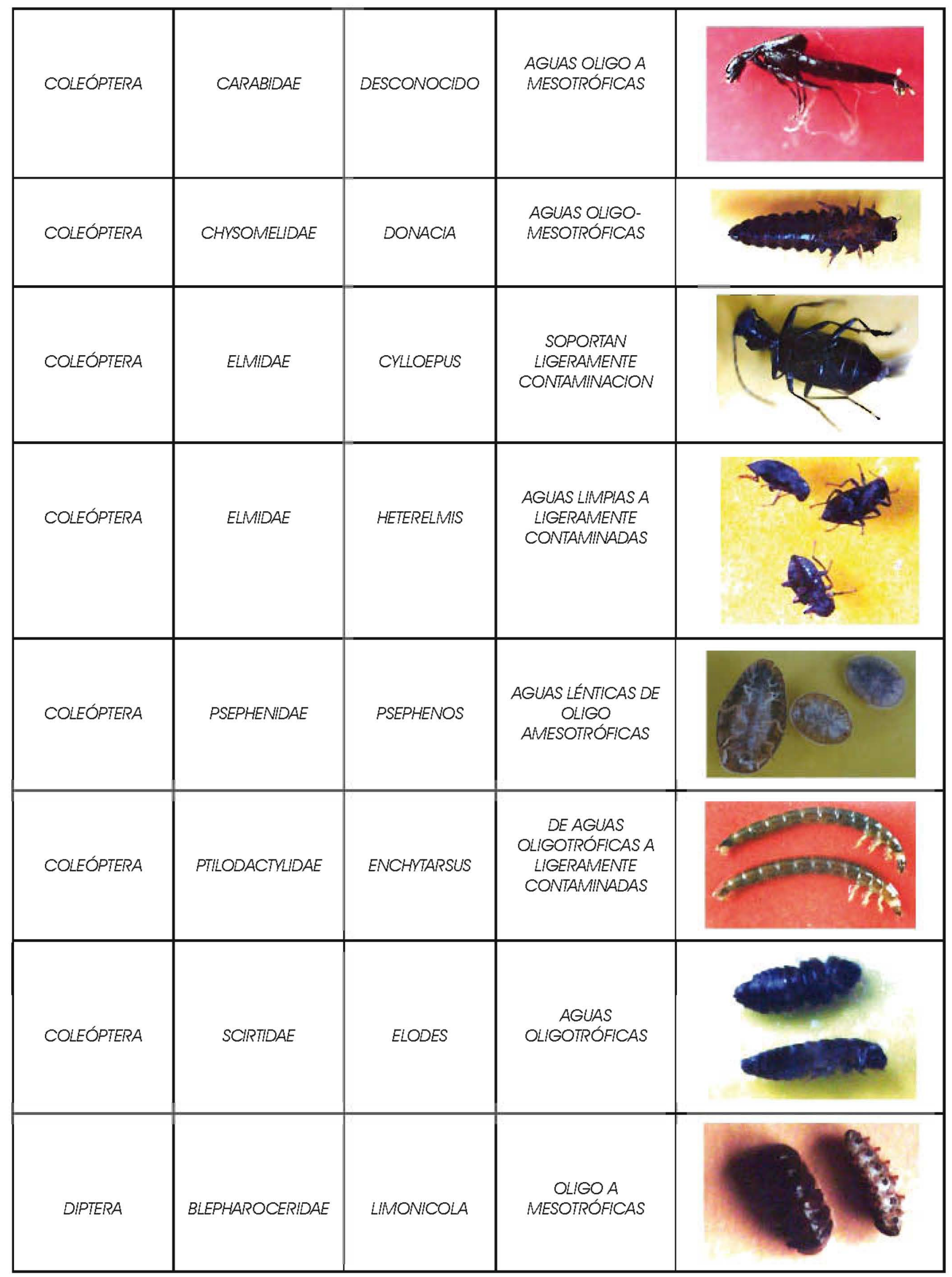




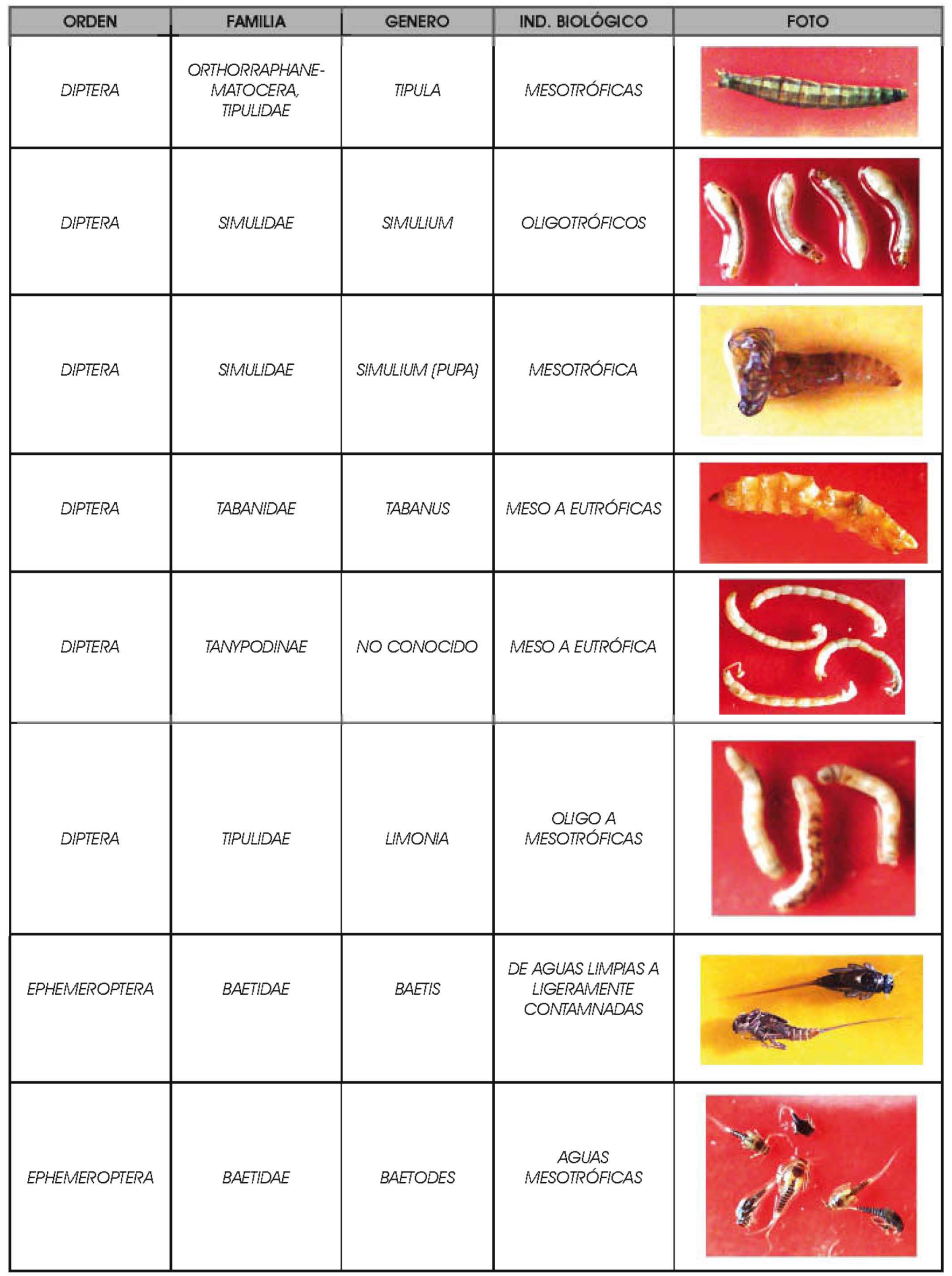




\begin{tabular}{|c|c|c|c|c|}
\hline ORDEN & FAMILIA & GENERO & IND. BIOLÓGICO & FOTO \\
\hline EPHEMEROPTERA & BAETIDAE & DACTYLOBAETIS & $\begin{array}{l}\text { AGUAS LIMPIAS A } \\
\text { LIGERAMENIE } \\
\text { CONTAMINADAS }\end{array}$ & \\
\hline EPHEMEROPTERA & LEPTOPHLEBIDAE & TRAULODES & $\begin{array}{c}\text { AGUAS } \\
\text { OLIGOTRÓFICAS }\end{array}$ & \\
\hline EPHEMEROPTERA & TRICORYTIDAE & LEPTOHMPES & $\begin{array}{l}\text { AGUAS AGUA } \\
\text { MESOTRÓ }\end{array}$ & \\
\hline EPHEMEROPTERA & TRICORYTIDAE & TRICORYTHODES & $\begin{array}{c}\text { AGUA LIGERAMENTE } \\
\text { CONTAMINADA }\end{array}$ & \\
\hline $\begin{array}{c}\text { GLOSSIPHONMOR- } \\
\text { FA }\end{array}$ & GLOSSIFONIIDAE & PLACOBDELLA & $\begin{array}{c}\text { AGUAS } \\
\text { CONTAMINADAS }\end{array}$ & \\
\hline HAPLOTAIDA & HAPLOTAXIDAE & DESONOCIDO & $\begin{array}{c}\text { AGUAS } \\
\text { CONTAMINADAS }\end{array}$ & \\
\hline HAPLOTAXIDA & TUBIFICIDAE & TUBIFEX & $\begin{array}{l}\text { ACENTUADA } \\
\text { CONIAMINACIÓN } \\
\text { ACUATICA }\end{array}$ & \\
\hline HEMIPTERA & $\begin{array}{c}\text { GUERROMORPHA } \\
\text { VELHDAE }\end{array}$ & MICROVELIA & EURIALINAS & \\
\hline
\end{tabular}




\begin{tabular}{|c|c|c|c|c|}
\hline ORDEN & FAMILIA & GENERO & IND. BIOLÓGICO & FOTO \\
\hline LEPIDOPTERA & PYRALIDAE & CATACLYSTA & OLIGOTRÓFICAS & \\
\hline ODONATA & AESHNIDAE & AESHNA & MESOTRÓFICAS & \\
\hline ODONATA & COENAGRIONIDAE & ARGIA & $\begin{array}{c}\text { OLIGOMESOTRÓFH- } \\
\text { CAS }\end{array}$ & \\
\hline PLECOPTERA & PERLIDAE & ANACRONEURIA & OLIGOTRÓFICAS & \\
\hline TRICLADIDA & PLANARIDAE & DUGESIA & OLGOTRÓFICAS & \\
\hline TRICHOPTERA & GLOSSOSOMATIDAE & MOTONELLA & $\begin{array}{c}\text { OLGOA A } \\
\text { MESOIRÓFICA }\end{array}$ & \\
\hline TRICHOPTERA & HELICOPSYCHIDAE & HELICOPSYCHE & $\begin{array}{c}\text { OLIGOA } \\
\text { MESOTÓFICAS }\end{array}$ & \\
\hline TRICHOPTERA & HVDROPSYCHIDAE & ATOPSYCHE & $\begin{array}{c}\text { OLIGOA A } \\
\text { MESOTRÓFICA }\end{array}$ & \\
\hline
\end{tabular}




\begin{tabular}{|c|c|c|c|c|}
\hline ORDEN & FAMILIA & GENERO & IND. BIOLÓGICO & FOTO \\
\hline TRICHOPTERA & HVDROPSYCHIDAE & LEPTONEMA & $\begin{array}{c}\text { OLIGOA A } \\
\text { MESOTRÓFICA }\end{array}$ & \\
\hline TRICHOPTERA & LEPTOCERIDAE & ATANATOLICA & OLIGOTRÓFICA & \\
\hline TRICHOPTERA & ODONTOCERIDAE & MARILIA (PUPA) & AGUAMESOTRÓFICA & \\
\hline TRICHOPTERA & PHILOPOTAMIDAE & CHMARRA & OLIGOTRÓFICAS & \\
\hline TRICHOPTERA & POLIENTROPODINAE & POLICENTROPUS & $\begin{array}{c}\text { OLIGOMESOTROPI- } \\
\text { CAS }\end{array}$ & \\
\hline
\end{tabular}

